\title{
An Account of Cloned Genes of Methyl-erythritol-4- phosphate Pathway of Isoprenoid Biosynthesis in Plants
}

\author{
Deepak Ganjewala ${ }^{1 *}$, Shiv Kumar ${ }^{1}$, and Rajesh \\ Luthra $^{2}$
}

${ }^{1}$ School of Biotechnology, Chemical and Biomedical Engineering, VIT University, Vellore-632 014 (T.N.), India ${ }^{2}$ Human Resource Development Group, Council of Scientific and Industrial Research (CSIR), Pusa, New Delhi-110 012, India

Received 8 August 2008

Revised 19 September 2008

Accepted 23 October 2008

\begin{abstract}
:
Isoprenoids, also known as terpenoids, are biosynthesized by the condensation of the two $\mathrm{C}_{5}$ unit isopentenyl diphosphate (IPP) and isomer dimethylallyl diphosphate (DMAPP). Generally, plants use two separate pathways plastidial Methyl-erythritol-4-phosphate (MEP) and cytosolic acetate-mevalonate (MVA) pathways for formation of IPP. The genes, enzymes and intermediates of the MEP pathway have been unravelled in plants over the past few years. Interestingly, MEP pathway enzymes are encoded by nuclear genes but they function in plastids to produce precursors for isoprenes, monoterpenes, carotenoids, abscisic acid, gibberellins, and the side chain of chlorophylls, tocopherols, phylloquinones, and plastoquinone. In Arabidopsis thaliana, a complete set of genes of MEP pathway homologous to the $E$. coli MEP pathway genes have been identified. Although, these genes have been cloned and characterized from several other plants but overall information about them at one place is not available so far. Though, a range of reviews are available about their roles in isoprenoid biosynthesis and regulation. Therefore, we decided to compile the data on cloned and characterized genes of MEP pathway in plants. Also, we summarize the results of the previously published reports, particularly those which were based on incorporation of ${ }^{13} \mathrm{C}$-glucose or by application of specific inhibitors such as mevinolin and fosmidomycin to look into the MEP pathway in plants. In addition, we searched for the two key enzymes DXS and HMGR that could be assigned for the acetate-MVA and MEP pathway with the help of bioinformatics tools. Presence or absence of these enzymes can be correlated with respective isoprenoid biosynthetic pathways in plants.
\end{abstract}

*For correspondence: deepakganjawala73@yahoo.com Tel: +91-416-2243093; Fax: +91-416-2243091

Cell: $+91-99945-47146$
Keywords: Isoprenoids; Methyl-erythritol-4-phosphate (MEP); Acetate-MVA pathway; Hydroxyl-methylglutarylCoA reductase (HMGR); 1-deoxy-D-xylulose 5-phosphate synthase (DXPS or DXS).

\section{Introduction}

Plant secondary metabolites viz., isoprenoids have always been fascinating for the researchers. Obviously, they are the largest and most diverse class of plant secondary metabolites (Dubey et al., 2003). Over the years isoprenoids have been exhaustively investigated. There are many research papers, reviews, commentaries and books are available covering various aspects of isoprenoids viz., biosynthesis and regulation and functions in plants and microorganisms (Croteau, 1987; Rohmer, 1999; 2003; Lichtenthaler, 1999; Lichtenthaler, 2001; Eisenreich et al., 1997; 2001; 2004; RodrıguezConcepcion and Boronat, 2002; Chemler et al., 2006; Cheng et al., 2007). Isoprenoids are multifunctional; they play very important roles in membrane structure, redox reactions, light harvesting and photo-protection, and regulations of growth and development. They not only perform various roles in the plant's life such as, plantenvironment, plant-insect, plant-microorganism and plant-plant interactions but they became an essential part of our life as medicines, flavours, fragrances, cosmetics, dyes, insecticides and more (Verpoorte et al., 2002; Harborne, 2001; Dixon, 2001). Currently, isoprenoids are being used as anti-cancer and antimicrobial drugs for example artimicinin as a powerful antimalarial (Dhingra et al., 2000) and taxol as anti-cancer (Cragg et al., 1997) agents.

Despite the great deal of structural and functional diversity all isoprenoids are synthesized by consecutive condensation of common $\mathrm{C}_{5}$ isoprene precursor; isopentenyl diphosphate (IPP) and its isomer dimethylallyl diphosphate (DMAPP). IPP therefore is regarded as the universal precursor of all the isoprenoids. IPP, in turn is biosynthesized in two different sites via two separate and independent biochemical pathways: 1. Cytosolic acetate-MVA pathway and 2. Plastidial Methylerythritol 4- phosphate (MEP) also called as 1-deoxy-Dxylulose 5-phosphate (DOXP) or glyceraldehyde-3phosphate-pyruvate (GAP-Pyruvate) pathway (Fig. 1). In the present article, the pathway is described as MEP pathway. In general, acetate-MVA pathway produces sesquiterpenes and triterpenes while MEP pathway produces monoterpenes, sesquiterpenes, diterpenes, tetraterpenes, plastoquinone and prenyl side chains of chlorophyll.

In brief, MEP pathway (Fig. 1) begins with the formation of 1-deoxy-D-xylulose 5-phosphate (DOXP/ DXP) by the condensation of pyruvate and glyceraldehyde 3-phosphate catalysed by DOXP synthase (DXS, 


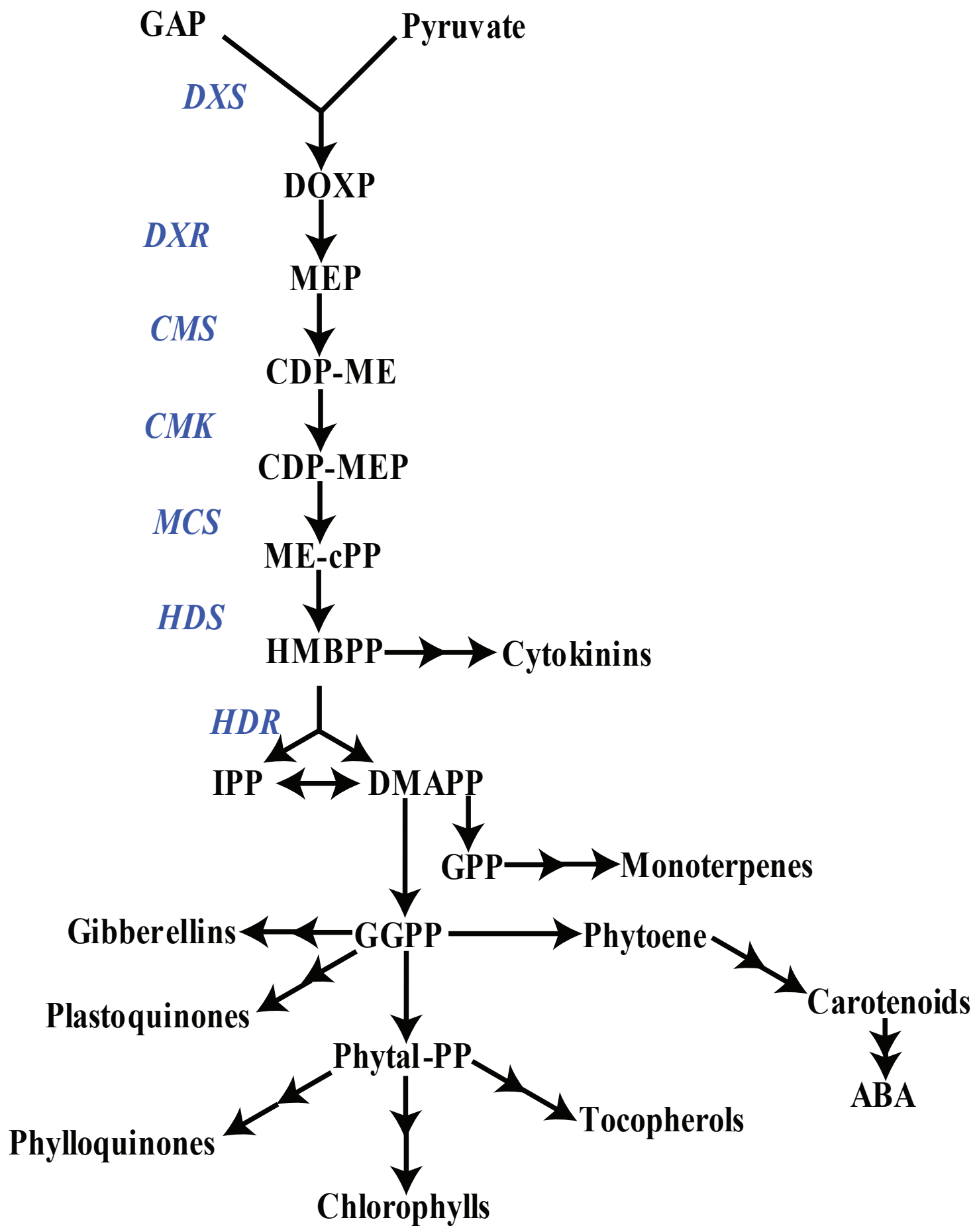

Fig. 1. MEP pathway for the biosynthesis of isoprenoids in plants. ABA, abscisic acid; CDP-ME, 4-(cytidine-5'-diphospho)-2-C-methyl-D-Erythritol; CDP-MEP, 4-Diphosphocytidyl-2C-methyl-D-erythritol 4-phosphate; DMAPP, dimethylallyl diphosphate; DXP, 1-deoxy-D-xylulose 5-phosphate; GAP, glyceraldehyde 3-phosphate; GGPP, geranylgeranyl diphosphate; GPP, geranyl diphosphate; HBMPP, 4-hydroxy-3-methylbut-2-enyl diphosphate; IPP, isopentenyl diphosphate; ME-cPP, 2C-methyl-D-erythritol 2,4-cyclodiphosphate; MEP, 2C-methylerythritol 4-phosphate. Enzymes are indicated in bold italic; 4-(cytidine-5'-diphospho)-2-C-methyl-D-Erythritol kinase (CMK, EC 2.7.1.148); 4-Diphosphocytidyl-2C-methyl-D-erythritol 4-phosphate synthase (CMS, EC 2.7.7.60); 1-deoxy-D-xylulose 5-phosphate reductoisomerase (EC 1.1.1.267 ); 1-deoxy-D-xylulose 5-phosphate synthase (DXS, EC 4.1.3.37); 4-hydroxy-3-methylbut-2-enyl diphosphate reductase (HDR, EC 1.17.1.2 ) ; 4-Hydroxy-3-methylbut2-en-yl-diphosphate synthase (HDS, EC, 1.17.4.3); 2C-methyl-D-erythritol 2,4-cyclodiphosphate synthase (MCS, EC 4.6.1.12). 
(EC 4.1.3.37). DOXP then undergo intra-molecular rearrangement and reduction catalysed by 1-deoxyD-xylulose 5-phosphate reductoisomerase (DXR, EC 1.1.1.267) to yield methyl erythritol-4-phosphate (MEP) which is regarded as an immediate precursor of plastidic isoprenoids. MEP is consecutively converted into 4-diphosphocytidyl-methylerythritol (CDP-ME), 4-diphosphocytidyl-methylerythritol (CDP-MEP) and methyl-erythritol 2,4-cyclodiphosphate (ME-cPP). These reactions are carried out by CDP-ME synthase (CMS, EC 2.7.7.60), CDP-ME kinase (CMK, EC 2.7.1.148) and ME-cPP synthase (MCS, EC 4.6.1.12). Methylerythritol 2,4-cyclodiphosphate (ME-cPP) then converted to hydroxymethylbutenyl 4-diphosphate (HMBPP) by an enzyme hydroxymethylbutenyl 4-diphosphate synthase (HDS, EC 1.17.4.3). HMBPP is finally converted into a mixture of IPP and DMAPP by the enzyme HMBPP reductase (HDR, 1.17.1.2) (Eisenreich et al., 2001; 2004).

At present, the genes encoding enzymes of MEP pathway with homology to the E. coli MEP pathway enzymes haven been identified from variety of plants including Arabdopsis thaliana (http://www.Arabidopsis. org). The present review is aimed to provide the information on these cloned and characterized genes of MEP pathway in plants. Basically, the idea to compile the information on MEP pathway genes came from a very likely article on cloned genes of acetate-MVA pathway in plants by Scolink and Bartley (1996). The information about cloned genes of MEP pathway in one piece would be helpful to home in to isoprenoid biosynthesis. The results presented here were derived through online search and analysis (http://www.ncbi.nlm.nih.gov/; http:// www.tigr.org/). In addition, published literatures were also taken in account such as those describe application of ${ }^{13} \mathrm{C}$-glucose-Nuclear Magnetic Resonance (NMR) spectroscopy for elucidation of MEP pathway in plants. In addition, we took the help of bioinformatics tools to search DXS and HMGR, two key enzymes of their respective pathways to investigate these pathways in plants.

\section{${ }^{13} \mathrm{C}$-glucose-NMR spectroscopy provides clues for the MEP pathway}

The potential of using ${ }^{13} \mathrm{C}$-glucose-NMR spectroscopy to elucidate metabolic pathways in plants has long been recognized. Early efforts relied on NMR spectra of metabolites which were related to the underlying pathways used to create them (Jeffrey et al., 1991). NMR spectra have also been used to elucidate the flux through metabolic pathways (Bacher et al., 1998; Kelleher, 2001). The use of in vivo ${ }^{13} \mathrm{C}-\mathrm{NMR}$ spectroscopy to study the biosynthesis of secondary metabolites in plants has been well documented previously (see Table 2). Useful clues to the origin of the carbon atoms of isoprenoids in bacteria and for the elucidation of the MVA independent route were obtained from labeling experiments using ${ }^{13} \mathrm{C}$-glucose with Zymomonas mobilis, a facultative anaerobic and fermentative bacterium (Sprenger, 1996). The use of NMR spectroscopy in plant secondary metabolism has been hampered mainly because of very low concentration of the secondary metabolites, and the pathway leading to isoprenoid formation are often branched hence the
${ }^{13} \mathrm{C}$-label of early precursors is diluted into several metabolites at the end. These difficulties in resolving the origin of Isoprenoid units could be overcome by NMR analysis of extracts or isolated compounds. To investigate the biosynthetic origin of isoprenoid building blocks of secondary metabolites, the pathway-independent precursor ${ }^{13} \mathrm{C}$-glucose, which produces distinctly different labelling patterns of the individual isoprene units for the MEP and MVA pathways is generally employed (Rohmer, 1999). Given that glucose is a general intermediary metabolite, the isotope from the proffered carbohydrate can be diverted to virtually all metabolic compartments and intermediates in plant cells (Eisenreich et al., 2004).

The biosynthetic origin of a considerable number of primary and secondary plant terpenoids has been and currently being reinvestigated using ${ }^{13} \mathrm{C}$-glucoseNMR spectroscopic technique in higher and lower plants (liverworts). Table 1 provides the information on application of ${ }^{13} \mathrm{C}$-NMR spectroscopy to elucidate the MEP pathway in plants. The data show that a wide variety of monoterpenes, diterpenes and sesquiterpenes (germacrene) are biosynthesized predominantly via the MEP pathway. Beside the analysis of these published reports on MEP pathways, our online database search for DXS and HMGR the key regulatory enzyme respectively of MEP and acetate-MVA pathways further provided the clue for the operation of either of these pathways in plants. Table 2 provides information on distribution of DXS and HMGR enzymes in plants. This information was collected online from http://www.ncbi.nlm.nih.gov/.

\section{Cloned genes of MEP pathway}

Our knowledge and understanding about the biosynthesis and regulation of isoprenoids in plants has been tremendously increased during the past two decades. As a result, genes encoding enzymes of the MEP pathway have been cloned and characterized from a several plants in the recent time. Though, several genes of the MEP pathway downstream from ispC were discovered by a strategy combining biochemical evidence with comparative genomic analysis. Please see a review by Eisenreich et al. (2004) for detailed description about the mechanism of action of enzymes of MEP pathway. Here in the Table 3 we provide information exclusive on cloned and characterized genes of the MEP pathway in plants. The information mainly comprises of GeneBank accession number, size and protein or gene name. Table 3 shows that DXS and DXR have been cloned and characterized from a variety of plants while other enzymes of the MEP pathway could only be characterized only from a few plants. So far, HDS is known from only two plants Nicotiana benthamiana and Oryza sativa. Similarly HDR is also known from only two plants, Arabidopsis thaliana and $N$. benthamiana. An overall distribution of MEP genes in plants is presented in Table 4. From Table 4 , it is clear that $A$. thaliana and $O$. sativa (Japonica var.) genome has complete set of genes encoding enzymes of MEP pathway along with HMGR of acetate-MVA pathway. Stevia rebaudiana genome has shown at least six of the seven genes of MEP pathway, but lacks the HMGR of acetate-MVA pathway. Further analysis of the data has revealed that 19 out of 39 plants searched online have 
Table 1. MEP pathway in plants verified on the basis of ${ }^{13} \mathrm{C}$-glucose-NMR spectroscopy.

\begin{tabular}{|c|c|c|}
\hline Plants & Isoprenoids & References \\
\hline Catharanthus roseus & $\begin{array}{l}\text { Terpenoids } \\
\text { Iridoid glucoside secologanin }\end{array}$ & Arigoni et al., 1997; 1999; Contin et al., 1998 \\
\hline $\begin{array}{l}\text { Chelidonium majus } \\
\text { Populus nigra } \\
\text { Salix viminalis }\end{array}$ & Isoprene & Zeidler et al., 1997, 1998 \\
\hline Conocephalum conicum & Isoprenoids & Thiel et al., 2002 \\
\hline $\begin{array}{l}\text { Dacus carota } \\
\text { Hordeum vulgare } \\
\text { Lemna gibba }\end{array}$ & L-carotene, lutein, prenyl chains of chlorophylls and plastoquinone-9 & Lichtenthaler et al., 1997 \\
\hline Eucalyptus globules & Cineol & Rieder et al., 2000 \\
\hline Fossombronia alaskana & Hopane triterpene and three diterpenes & Hertewich et al., 2001 \\
\hline Hordeum vulgare & sesquiterpenoid cyclohexane derivatives & Maier et al., 1998 \\
\hline Liriodendron tulipifera & Terpenes & Sagner et al., 1998 \\
\hline Marrubium vulgare & Labdane diterpenoid marrubin & Knoss et al., 1997 \\
\hline Matricaria recutita & Isoprene units of chamomile sesquiterpenes & Adam and Zapp, 1998 \\
\hline Mentha citrate & Linalyl Acetate monoterpene & Fowler et al., 1999 \\
\hline Mentha pulegium & Monoterpenes & Eisenreich et al., 1997 \\
\hline Narcissus pseudonarcissus & $b$-carotene & Fellermeier et al., 1999 \\
\hline $\begin{array}{l}\text { Pelargonium graveolens } \\
\text { Thymus vulgaris }\end{array}$ & Monoterpenes & Eisenreich et al., 1997 \\
\hline Persea Americana & Abscisic acid; Carotenoids and abscisic & Hirai et al., 2000; Milborrow et al., 1998 \\
\hline Rauwolfia serpentine & Monoterpene loganin & Eichinger et al., 1996 \\
\hline Taxus chinensis & Taxol (diterpene) & Eisenreich et al., 1996 \\
\hline Trichcolea tometella & $\begin{array}{l}\text { Trichcolein and deoxytometellin (Hemi- and mono- terpene moieties } \\
\text { and diterpene phytol) }\end{array}$ & Barlowa et al., 2003 \\
\hline Vitis Vinifera & Linalool and geraniol & Klink et al., 2005 \\
\hline Lepidolaena hodgsoniae & Sesquiterpene hodgsonox & Luan et al., 2002 \\
\hline Anisotome layallii & Anisotomenes (bicyclic irregular diterpenes) & Barlowa et al., 2003 \\
\hline Piper aduncum & Isoprene Units in Chromenes & Leite et al., 2007 \\
\hline Solidago Canadensis & Germacrene D (sesquiterpenes) & Steliopoulos et al., 2002 \\
\hline
\end{tabular}

genes of both MEP and acetate-MVA pathway in their genome, while the others 20 plants had exclusively genes of MEP pathway.

\section{Conclusion}

Many pathogenic microorganisms including Mycobacterium tuberculosis and Plasmodium falciparum also operate MEP pathway for the biosynthesis of isoprenoids. In fact, isoprenoids plays crucial role in the survival of $P$. falciparum in host cells. Knowledge of the MEP pathway in such pathogenic microorganism is currently being exploited for the development of structurebased anti-microbial drugs by targeting the enzymes of MEP pathway. Therefore, details concerning the genes, enzymes and intermediates of the MEP pathway have become essential in achieving these goals. Currently, fosmidomycin an inhibitor of DOXP reductoisomerase (DXR) of MEP pathway has been successfully tested to hang-up isoprenoid biosynthesis in P. falciparum. Similar strategies could be employed for the development of novel herbicides (Lichtenthaler et al., 2000). This aspect of the isoprenoids researches have a direct impact on human health, hence created much interest and awareness among the researchers in the recent years to look for new structure based drugs against more pathogenic microorganisms and weeds relying on MEP pathway. Our, knowledge and understanding about the plant secondary metabolite biosynthesis and regulation has greatly accelerated these efforts. Most certainly, comparative genomics and in combination of bioinformatics has been an aid.

\section{Acknowledgements}

Authors are grateful to the Chancellor, VIT University for providing the necessary support and Professor Lazar Mathew and Professor Braj Nandan Prasad, School of Biotechnology, Chemical and Biomedical Engineering, VIT University for their constant encouragement and valuable suggestions. Financial support from the Department of Sciences and Technology (DST) and Council of Scientific and Industrial Research (CSIR), New Delhi, Government of India is duly acknowledged. 
Table 2. Results of online search for DXS and HMGR. DXS is assigned to MEP whereas HMGR to acetate-MVA pathway.

\begin{tabular}{|c|c|c|c|c|}
\hline \multirow[b]{2}{*}{ Plants } & \multicolumn{2}{|c|}{$\begin{array}{l}\text { MEP Pathway } \\
\text { DOXP Synthase (DXS) }\end{array}$} & \multicolumn{2}{|c|}{$\begin{array}{l}\text { Acetate-Mevalonate Pathway } \\
\text { HMG-CoA Reductase (HMGR) }\end{array}$} \\
\hline & Accession No. & Size & Accession No. & Size \\
\hline Arabidopsis thaliana & BAB02345 & 604 & AAA67317 & 562 \\
\hline Antirrhinum majus & AAW28999 & 733 & & \\
\hline Artemisia annua & AAD56390 & 713 & AAD47596 & 567 \\
\hline Andrographis paniculata & AAP14353 & 691 & AAP14352 & 556 \\
\hline Camptotheca acuminate & & & AAB69726 & 575 \\
\hline Capsicum annuum & CAA75778 & 719 & AAD28179 & 604 \\
\hline Catharanthus roseus & CAA09804 & 716 & AAT52222 & 601 \\
\hline Cistus incanus subsp. creticus & & & ABL10110 & 388 \\
\hline Chrysanthemum x morifolium & BAE79547 & 669 & & \\
\hline Elaeis guineensis & AAS99588 & 707 & & \\
\hline Ginkgo biloba & AAS89341 & 717 & AAU89123 & 571 \\
\hline Hevea brasiliensis & AAS94123 & 720 & CAA38467 & 575 \\
\hline Lycopersicon esculentum & AAD38941 & 719 & AAB62581 & 601 \\
\hline Lycopersicon hirsutum & AAT97962 & 714 & & \\
\hline Mentha $x$ piperita & AAC33513 & 724 & & \\
\hline \multirow[t]{3}{*}{ Medicago truncatula } & ABP03805 & 711 & ABE88827 & 583 \\
\hline & ABP03804 & 710 & & \\
\hline & ABO82094 & 717 & & \\
\hline Morinda citrifolia & AAL32062 & 722 & & \\
\hline Narcissus pseudonarcissus & CAC08458 & 709 & & \\
\hline Nicotiana tabacum & & & AAB87727 & 604 \\
\hline Oryza sativa (Japonica) & NP_001055524 & 720 & BAD10066 & 561 \\
\hline \multirow[t]{2}{*}{ Oryza sativa (Indiaca) } & EAY98024 & 710 & CAA92821 & 576 \\
\hline & AAB88295 & 594 & & \\
\hline Pueraria montana var. lobata & AAQ84169 & 717 & & \\
\hline Picrorhiza kurrooa & & & $\mathrm{ABC} 74565$ & 561 \\
\hline \multirow[t]{2}{*}{ Salvia miltiorrhiza } & & & ABB45812 & 174 \\
\hline & & & AAU87798 & 267 \\
\hline Stevia rebaudiana & CAD22155 & 715 & & \\
\hline Tagetes erecta & AAG10432 & 725 & AAC15475 & 574 \\
\hline \multicolumn{5}{|l|}{ Taxus cuspidate } \\
\hline Taxus x media & AAS89342 & 742 & AAQ82685 & 595 \\
\hline \multirow[t]{4}{*}{ Zea mays } & ABP88134 & 719 & CAA70440 & 579 \\
\hline & ABP88135 & 705 & & \\
\hline & AAX49359 & 481 & & \\
\hline & AAX49358 & 424 & & \\
\hline Vitis Vinifera & CAN71054 & 1638 & CAN72217 & 575 \\
\hline
\end{tabular}

DOXP: 1-deoxy-D-xylulose 5-phosphate; HMG-CoA 3-hydroxy-3-methylglutaryl-coenzyme A. 
i40 Ganjewala et al.

Table 3. Cloned and characterized enzymes of the MEP pathway in plants.

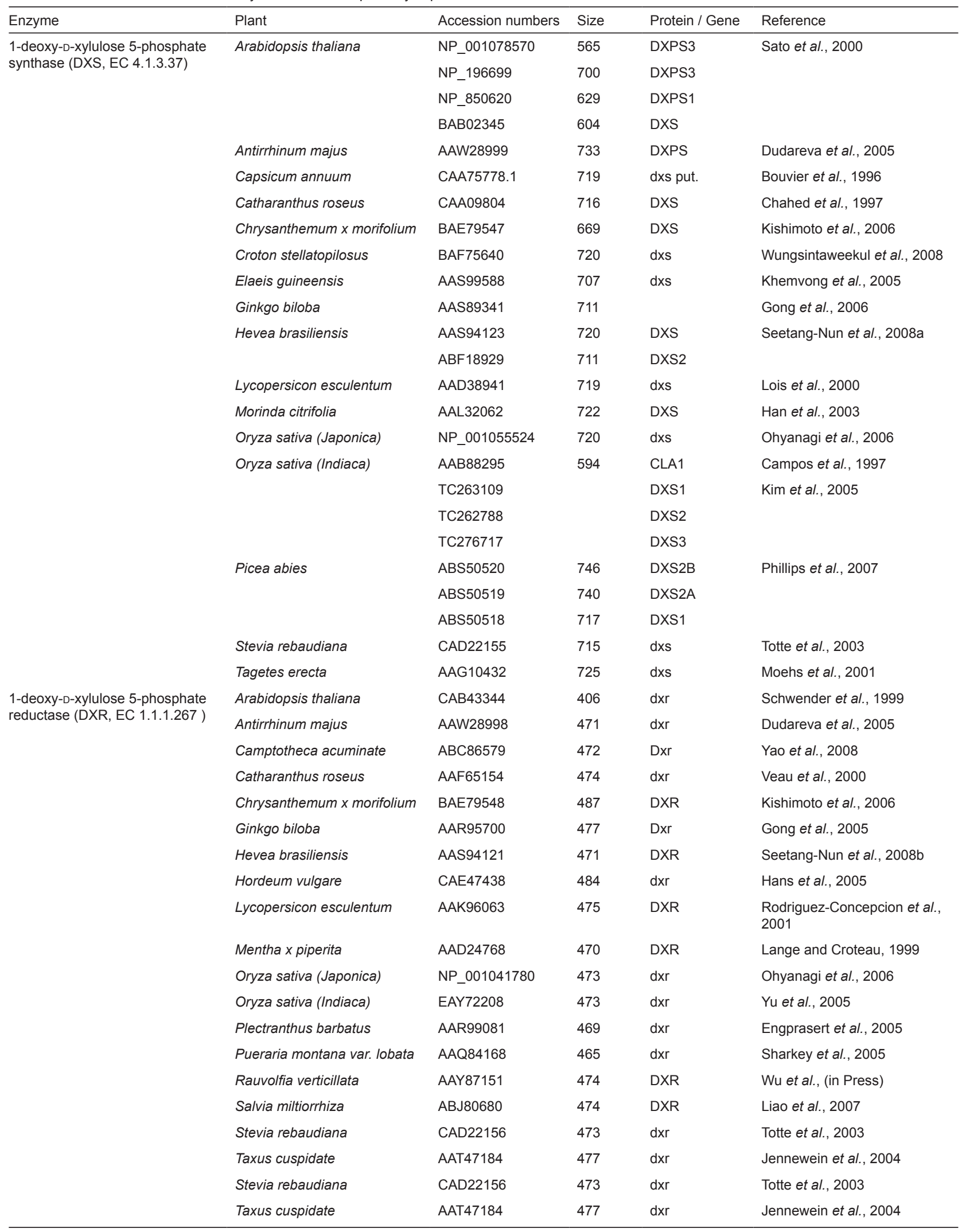


Table 3. Cloned

\begin{tabular}{|c|c|c|c|c|c|}
\hline Enzyme & Plant & Accession numbers & Size & Protein / Gene & Reference \\
\hline \multirow{5}{*}{$\begin{array}{l}\text { 4-Diphosphocytidyl-2C-methyl-D- } \\
\text { erythritol 4-phosphate synthase } \\
\text { (CMS, EC 2.7.7.60) }\end{array}$} & Arabidopsis thaliana & NP_565286 & 302 & ISPD & \multirow[t]{2}{*}{ Seki et al., 2002} \\
\hline & & BAC42737 & 302 & ispD & \\
\hline & Ginkgo biloba & AAZ80386 & 327 & MECT & Kim et al., 2005 \\
\hline & Oryza sativa (Japonica) & BAD82130 & 297 & ispD put. & \multirow{2}{*}{$\begin{array}{l}\text { Sasaki and Matsumoto, 2002; } \\
\text { Yu et al., } 2005\end{array}$} \\
\hline & Oryza sativa (Indiaca) & EAY76759 & 408 & ispD pat. & \\
\hline \multirow{4}{*}{$\begin{array}{l}\text { 4-(cytidine-5'-diphospho)-2-C- } \\
\text { methyl-D-Erythritol kinase (CMK, } \\
\text { EC 2.7.1.148) }\end{array}$} & Arabidopsis thaliana & O81014 & 383 & ISPE & Lin and Kaul, 1999 \\
\hline & Lycopersicon esculentum & AAF87717 & 401 & ispE & Lange and Croteau, 1999 \\
\hline & Mentha x piperita & P56848 & 405 & ISPE & Rohdich et al., 2000 \\
\hline & Oryza sativa (Japonica) & NP_001044544 & 401 & ispE & Ohyanagi et al., 2006 \\
\hline \multirow{5}{*}{$\begin{array}{l}\text { 2C-methyl-D-erythritol 2,4-cyclodi- } \\
\text { phosphate synthase (MCS, EC } \\
\text { 4.6.1.12) }\end{array}$} & Arabidopsis thaliana & AAM62786 & 231 & MECDP_S & Gao et al., 2006 \\
\hline & Ginkgo biloba. & AAY40863 & 239 & Mecps & Alexandrov et al., 2006 \\
\hline & Oryza sativa (Japonica) & EAZ24186 & 222 & MECDP_S & Yu et al., 2005 \\
\hline & Oryza sativa (Indica) & EAY87077 & 222 & MECDP_S & Yu et al., 2005 \\
\hline & Taxus $x$ media & ABB88956 & 247 & mecs & Jin et al., 2006 \\
\hline \multirow{2}{*}{$\begin{array}{l}\text { 4-Hydroxy-3-methylbut2-en-yl- } \\
\text { diphosphate synthase (HDS, EC, } \\
1.17 .4 .3 \text { ) }\end{array}$} & Nicotiana benthamiana & AAS75817 & 268 & gcpE/ispG & Page et al., 2003 \\
\hline & Oryza sativa (Japonica) & AAO72576 & 608 & gcpE & Cooper et al., 2003 \\
\hline \multirow{2}{*}{$\begin{array}{l}\text { 1-Hydroxy-2-methyl-butenyl } \\
\text { 4-diphosphate reductase (HDR, } \\
\text { EC 1.17.1.2 ) Or, 4-hydroxy-3- } \\
\text { methylbut-2-enyl diphosphate } \\
\text { reductase }\end{array}$} & Arabidopsis thaliana & AAW82381 & 468 & HDR/ISPH & Guevara-Garcia et al., 2005 \\
\hline & Nicotiana benthamiana & AAS75818 & 166 & ispH LytB & Page et al., 2003 \\
\hline
\end{tabular}

\section{References}

Adam, K.P. and Zapp, J. (1998) Biosynthesis of the isoprene units of chamomile sesquiterpenes. Phytochemistry 48, 953-959.

Alexandrov, N.N., Troukhan. M.E., Brover, V.V., Tatarinova, T., Flavell, R.B., and Feldmann, K.A. (2006) Features of Arabidopsis genes and genome discovered using fulllength cDNAs. Plant Mol. Biol. 60, 71-87, 2006.

Arigoni, D., Eisenreich, W., Latzel, C., Sagner, S., Radykewicz, T., Zenk, M.H., and Bacher, A. (1999) Dimethylallyl pyrophosphate is not the committed precursor of isopentenyl pyrophosphate during terpenoid biosynthesis from 1-deoxyxylulose in higher plants. Proc. Natl. Acad. Sci. U.S.A. 96, 1309-1314.

Arigoni, D., Sagner, S., Latzel, C., Eisenreich, W., Bacher, A., and Zenk, M.H. (1997) Terpenoid biosynthesis from 1-deoxy-Dxylulose in higher plants by intramolecular skeletal rearrangement. Proc. Natl. Acad. Sci. U.S.A. 94, 10600-10605.

Bacher,A., Rieder, C., Eichinger, D., Arigoni, D., Fuchs, G., Eisenreich,W. (1998) Elucidation of novel biosynthetic pathways and metabolite flux patterns by retrobiosynthetic NMR analysis. FEMS Microbiol. Rev. 22, 567-598.

Barlowa, A.J., Lorimer, S.D., Morgan, E.R., and Weavers, R.T. (2003) Biosynthesis of the sesquiterpene hodgsonox from the New Zealand liverwort Lepidolaena hodgsoniae. Phytochemistry 63, 25-29.

Bouvier, F., d'Harlingue, A., Suire, C., Backhaus, R.A., and Camara, B. (1998) Dedicated roles of plastid transketolases during the early onset of isoprenoid biogenesis in pepper fruits. Plant Physiol. 117, 14231431.

Campos, N., Lois, L.M., and Boronat, A. (1997) Nucleotide sequence of a rice cDNA encoding a transketolase-like protein homologous to the Arabidopsis CLA1 gene product. Plant Physiol. 115, 1289-1293.

Chahed, K., Oudin, A., Guivarch, N., Hamdi, S., Chenieux, J.C., Rideau, M., and Clastre, M. (2000) 1-DeoxyD-xylulose 5-phosphate synthase from periwinkle: cDNA identification and induced gene expression in terpenoidindole alkaloid-producing cells. Plant Physiol. Biochem. 38, 559-566.

Chemler, J.A., Yan, Y., and Koffas, M.K.G. (2006) Biosynthesis of isoprenoids, polyunsaturated fatty acids and flavonoids in Saccharomyces cerevisiae. Microb. Cell Fact. 5, 20-30.

Cheng, A.X., Lou, Y.G., Mao,Y.B., Lu, S., Wang, L.J., and Chen, X.Y. (2007) Plant Terpenoids: Biosynthesis and Ecological Functions. J. Integrative Plant Biol. 49, 179-186.

Contin, A., Vander-Heijden, R., Lefeber, A.W.M., and Verpoorte, R. (1998) The iridoid glucoside secologanin is derived from the novel triose-phosphate/pyruvate pathway in Catharanthus roseus cell culture. FEBS Lett. 434, 413-416.

Cooper, B., Clarke, J., Budworth, P., Kreps, J., Hutchison, D., Park, S., Guimil, S., Dunn, M., Luginbuhl, P., Ellero, C., Goff, S.A., and Glazebrook, J. (2003) A network of rice genes associated with stress response and seed development. Proc. Natl. Acad. Sci. U.S.A. 100, 49454950. 
Table 4: Overall distribution of MEP pathway genes in plants.

\begin{tabular}{|c|c|c|c|c|c|c|c|c|}
\hline \multirow[b]{2}{*}{ Plants } & \multicolumn{8}{|c|}{ MEP pathway genes } \\
\hline & $d x s$ & $y a e M / d x r$ & ygbP/mect/ispD & ychB/cmek/ispE & $y g b B / m e c s$ & $g c p E / i s p G$ & $h d r / i s p H$ & $\mathrm{Hmgr}$ \\
\hline Arabidopsis thaliana & + & + & + & + & + & + & + & + \\
\hline Andrographis paniculata & + & - & - & - & - & - & - & + \\
\hline Antirrhinum majus & + & + & - & - & - & - & - & - \\
\hline Artemisia annua & + & + & - & - & - & - & - & + \\
\hline Catharanthus roseus & + & + & - & - & + & - & - & + \\
\hline Camptotheca acuminate & & + & & & & & & + \\
\hline Cistus incanus subsp. creticus & & + & & & & & & + \\
\hline Chrysanthemum $x$ morifolium & + & + & - & - & - & - & - & - \\
\hline Citrus jambhiri & & & & & & + & & $?$ \\
\hline Croton stellatopilosus & + & + & - & - & - & - & - & - \\
\hline Elaeis guineensis & + & + & - & - & - & - & - & - \\
\hline Forsythia $x$ intermedia & + & + & - & + & - & - & + & - \\
\hline Ginkgo biloba & + & + & + & + & - & - & + & + \\
\hline Hevea brasiliensis & + & + & - & & - & + & - & + \\
\hline Hordeum vulgare & & + & & & & & & - \\
\hline Linum usitatissimum & & + & & & & & & - \\
\hline Lycopersicon esculentum & + & + & - & + & + & - & - & + \\
\hline Lycopersicon hirsutum & + & - & - & + & - & - & - & - \\
\hline Mentha x piperita & + & + & - & + & - & - & - & - \\
\hline Medicago truncatula & + & - & - & - & - & - & - & + \\
\hline Mesostigma viride & & & & + & & & + & $?$ \\
\hline Morinda citrifolia & + & - & - & - & - & - & - & - \\
\hline Narcissus pseudonarcissus & + & - & - & - & - & - & - & - \\
\hline Nicotiana benthamiana & & & & + & + & & + & - \\
\hline Nicotiana tabacum & & + & & & & & & + \\
\hline Oryza sativa (Japonica) & + & + & + & + & + & + & + & + \\
\hline Oryza sativa (Indiaca) & + & + & + & - & - & + & - & + \\
\hline Picrorhiza kurrooa & & + & & + & & & & + \\
\hline Plectranthus barbatus & & + & & & & & & - \\
\hline Pueraria montana var. lobata & + & + & - & - & - & - & - & - \\
\hline Rauvolfia verticillata & & + & & + & & & & - \\
\hline Salvia miltiorrhiza & & + & + & + & & & & + \\
\hline Stevia rebaudiana & + & + & + & + & + & + & - & - \\
\hline Tagetes erecta & + & + & - & - & - & - & - & + \\
\hline Taxus chinensis & & + & & & & & & - \\
\hline Taxus cuspidate & & + & & & & & & - \\
\hline Taxus $x$ media & + & + & - & - & - & + & - & + \\
\hline Vitis Vinifera & & & + & & & & & + \\
\hline Zea mays & + & + & - & - & + & - & - & + \\
\hline
\end{tabular}

dxs: 1-deoxy-D-xylulose 5-phosphate synthase; $\boldsymbol{d x r}$ : 1-deoxy-D-xylulose 5-phosphate reductase; ispD: 4-diphosphocytidyl2C-methyl-D-erythritol synthase; ispE: 4-diphosphocytidyl 2C-methyl-D-erythritol kinase; mecs; $2 C$-methyl-D-erythritol 2,4-cyclodiphosphatesynthase; ispG: 1-hydroxy-2-methyl-2-(E)butenyl 4-diphosphate synthase; ispH: 1-hydroxy-2-methyl-2-(E)-butenyl 4-diphosphate reductase; hmgr: 3-hydroxy-3-methylglutaryl-CoA reductase.

Cragg, G.M., Newman, D.J., and Snader, K.M. (1997) Natural products in drug discovery and development. J. Nat. Prod. 60, 52-60.

Croteau, R. (1987) Biosynthesis and catabolism of monoterpenoids. Chem. Rev. 87, 929-954.
Dhingra, V., Rao, K.V., and Narasu, M.L. (2000) Current status of artemisinin and its derivatives as antimalaria drugs. Life Sci. 66, 279-300.

Dixon. R.A. (2001) Natural products and plant disease resistance. Nature 411, 843-847. 
Dubey, V.S., Bhalla, R., and Luthra, R. (2003) An overview of the non-mevalonate pathway for terpenoid biosynthesis in plants. J. Biosciences 28, 637-646.

Dudareva, N., Andersson, S., Orlova, I., Gatto, N., Reichel, M., Rhodes, D., Boland, W., and Gershenzon, J. (2005) The nonmevalonate pathway supports both monoterpene and sesquiterpene formation in snapdragon flowers. Proc. Natl. Acad. Sci. U.S.A. 102, 933-938.

Eichinger, D., Bacher, A., Zenk, M.H., and Eisenreich, W. (1999) Analysis of metabolic pathways via quantitative prediction of isotope labeling patterns: a retrobiosynthetic ${ }^{13} \mathrm{C}$ NMR study on the monoterpene loganin. Phytochemistry 51, 223-226.

Eisenreich, W., Menhard, B., Hylands, P.J., Zenk, M.H., and Bacher, A. (1996) Studies on the biosynthesis of taxol: The taxane carbon skeleton is not of mevalonoid origin. Proc. Natl. Acad. Sci. U.S.A. 93, 6431-6436.

Eisenreich, W., Rohdich, F., and Bacher, A. (2001) Deoxyxylulose phosphate pathway to terpenoids. Trends Plant Sci. 6, 78-84.

Eisenreich, W., Sagner, S., Zenk, M.H., and Bacher, A. (1997) Monoterpenoid essential oils are not of mevalonoid origin. Tetrahedron Lett. 38, 3889-389.

Eisenreicha, W., Bachera, A., Arigonib, D., and Rohdicha, F. (2004) Biosynthesis of isoprenoids via the nonmevalonate pathway. Cell Mol. Life Sci. 61, 14011426.

Engprasert, S., Shoyama, Y., and Taura, F. (2005) Molecular cloning, expression and characterization of recombinant 1-deoxy-D-xylulose-5-phosphate reductoisomerase from Coleus forskohlii Brig. Plant Sci. 169, 287-294.

Fellermeier, M., Kis, K., Sagner, S., Maier, U., Bacher, A., and Zenk, M.H. (1999) Cell-free conversion of 1-deoxyD-xylulose 5-phosphate and 2-C-methyl-D-erythritol 4-phosphate into $\beta$-carotene in higher plants and its inhibition by fosmidomycin. Tetrahedron Lett. 40, 27432746.

Fowler, D.J., Hamilton, J.T.G., Humphrey, A.J., and O'Hagan, D. (1999) Plant Terpene Biosynthesis. The Biosynthesis of Linalyl Acetate in Mentha citrate. Tetrahedron Lett. 40, 3803-3806.

Gao, S., Lin, J., Liu, X., Deng, Z., Li, Y., Sun, X., and Tang, K. (2006) Molecular Cloning, Characterization and Functional Analysis of a 2C-methyl-D-erythritol 2,4-cyclodiphosphate Synthase Gene from Ginkgo biloba. J. Biochem. Mol. Biol. 39, 502-510.

Gong, Y., Liao, Z., Chen, M., Zuo, K., Guo, L., Tan, Q., Huang, Z., Kai, G., Sun,.X., Tan, F., and Tang, K. (2005) Molecular cloning and characterization of a 1-deoxyD-xylulose 5-phosphate reductoisomerase gene from Ginkgo biloba. DNA Seq. 16, 111-120.

Gong, Y.F., Liao, Z.H., Guo, B.H., Sun, X.F., and Tang, K.X. (2006) Molecular cloning and expression profile analysis of Ginkgo biloba DXS gene encoding 1-deoxyD-xylulose 5-phosphate synthase, the first committed enzyme of the 2-C-methyl-D-erythritol 4-phosphate pathway. Planta Med. 72, 329-335.

Guevara-Garcia, A., San Roman, C., Arroyo, A., Cortes, M.E., de la Luz Gutierrez-Nava, M., and Leon, P. (2005) Characterization of the Arabidopsis clb6 mutant illustrates the importance of posttranscriptional regulation of the methyl-D-erythritol 4-phosphate pathway. Plant Cell 17, 628-643.

Han, Y.S., Roytrakul, S., Verberne, M.C., van der Heijden, R., Linthorst, H.J.M., and Verpoorte, R. (2003) Cloning of a cDNA encoding 1-deoxy-D-xylulose 5-phosphate synthase from Morinda citrifolia and analysis of its expression in relation to anthraquinone accumulation. Plant Sci. 164, 911-917.

Hans, J., Hause, B., Strack, D., and Walter, M.H. (2004) Cloning, characterization, and immunolocalization of a mycorrhiza-inducible 1-deoxy-D-xylulose 5-phosphate reductoisomerase in arbuscule-containing cells of maize. Plant Physiol.134, 614-624.

Harborne, J.B. (2001) Twenty-five years of chemical ecology. Nat. Prod. Rep. 18, 361-379.

Hertewich, U., Zapp, J., Becker, H., and Adam, K.P. (2001) Biosynthesis of a hopane triterpene and three diterpenes in the liverwort Fossombronia alaskana. Phytochemistry 7, 1049-1054.

Hirai, N., Yoshida, R., Todoroki, Y., and Ohigashi, H. (2000) Biosynthesis of abscisic acid by the non-mevalonate pathway in plants, and by the mevalonate pathway in fungi. Biosci. Biotechnol. Biochem. 64, 1448-1458.

Jeffrey, F.M., Rajagopal, A., Malloy, C.R., and Sherry, A.D. (1991) ${ }^{13}$ C-NMR: a simple yet comprehensive method for analysis of intermediary metabolism. Trends Biochem. Sci. 6, 5-10.

Jennewein, S., Wildung, M.R., Chau, M., Walker, K., and Croteau, R. (2004) Random sequencing of an induced Taxus cell cDNA library for identification of clones involved in Taxol biosynthesis. Proc. Natl. Acad. Sci. U.S.A. 101, 9149-9154.

Jin, H., Gong, Y., Guo, B., Qiu, C., Liu, D., Miao, Z., Sun, $X$., and Tang, K. (2006) Isolation and characterization of a 2C-methyl-D-erythritol 2,4-cyclodiphosphate synthase gene from Taxus media. Mol. Biol. (N.Y.) 40, 914-921.

Kelleher, J.K. (2001) Flux estimation using isotopic tracers: common ground for metabolic physiology and metabolic engineering. Metab. Eng. 3, 100-110.

Khemvong, S., and Suvachittanont, W. (2005) Molecular cloning and expression of a cDNA encoding 1-deoxy-Dxylulose-5-phosphate synthase from oil palm in Elaeis guineensis Jacq. Plant Sci. 169, 571-578.

Kim, S.M., Kuzuyama, T., Chang, Y.J., Kwon, H.J., and Kim, S.U. (2006) Cloning and functional characterization of 2-C-methyl-D-erythritol 4-phosphate cytidyltransferase (GbMECT) gene from Ginkgo biloba. Phytochemistry 67, 1435-1441.

Kishimoto, S., and Ohmiya, A. (2006) Regulation of carotenoid biosynthesis in petals and leaves of chrysanthemum (Chrysanthemum morifolium) Physiol. Plantarum. 128, 436-447.

Klink, J.W., Becker, H., and Perry, N.B. (2005) Biosynthesis of irregular diterpenes in Anisotome layallii by head to tail coupling of geranyl diphosphate. Org. Biol. Chem. 3, 542-545.

Knoss, W., Reuter, B., and Zapp, J. (1997) Biosynthesis of the labdane diterpenoid marrubin in Marrubium vulgare via a nonmevalonate pathway. Biochem. J. 326, 449-454. 
Lange, B.M., and Croteau, R. (1999) Isoprenoid biosynthesis via a mevalonate-independent pathway in plants: cloning and heterologous expression of 1-deoxy-D-xylulose-5-phosphate reductoisomerase from peppermint. Arch. Biochem. Biophys. 365, 170174.

Lange, B.M., and Croteau, R. (1999) Isopentenyl diphosphate biosynthesis via a mevalonate-independent pathway: isopentenyl monophosphate kinase catalyzes the terminal enzymatic step. Proc. Natl. Acad. Sci. U.S.A. 96, 13714-13719.

Leite, A.C., Lopes, A.A., Kato, M.J., Bolzania, V.S., and Furlan, M., (2007) Biosynthetic Origin of the Isoprene Units in Chromenes of Piper aduncum (Piperaceae) J. Braz. Chem. Soc. 18, 1500-1503.

Liao, Z., Chen, R., Chen, M., Yang, C., Wang, Q., and Gong, Y. (2007) A new 1-deoxy-D-xylulose 5-phosphate reductoisomerase gene encoding the committed-step enzyme in the MEP pathway from Rauvolfia verticillata. Z. Naturforsch C. J. Biosci. 62, 296-304.

Lichtenthaler, H.K. (2001) Non-mevalonate isoprenoid biosynthesis: enzymes, genes and inhibitors. Biochem. Soc. Transact. 28, 785-789.

Lichtenthaler, H.K., Schwender, J., Disch, A., and Rohmer, M. (1997) Biosynthesis of isoprenoids in higher plant chloroplasts proceeds via a mevalonate-independent pathway. FEBS Lett. 400, 271-274.

Lichtenthaler, H.K. (1999) The 1-deoxy-D-xylulose-5phosphate pathway of isoprenoid biosynthesis in plants. Ann. Rev. Plant Physiol. Plant Mol. Biol. 50, 47-65.

Lichtenthaler, H.K., Zeidler, J., Schwender, J., and Muller, C. (2000) The non-mevalonate isoprenoid biosynthesis of plants as a test system for new herbicides and drugs against pathogenic bacteria and the malaria parasite. Z. Naturforsch. 55, 305-313.

Lin, X., and Kaul, S. (1999) Sequence and analysis of chromosome 2 of the plant Arabidopsis thaliana. Nature 402, 761-768.

Lois, L.M., Rodriguez-Concepcion, M., Gallego, F., Campos, N., and Boronat, A. (2000) Carotenoid biosynthesis during tomato fruit development: regulatory role of 1-deoxy-D-xylulose 5-phosphate synthase. Plant J. 22, 503-513.

Luan, F., and Wust, M. (2002) Differential incorporation of 1-deoxy-D-xylulose into (3S)-linalool and geraniol in grape berry exocarp and mesocarp. Phytochemistry 60, 451-459.

Maier, W., Schneider, B., and Strack, D. (1998) Biosynthesis of sesquiterpenoid cyclohexane derivatives in mycorrhizal barley roots proceeds via the glyceraldehydes-3-phosphate/pyruvate pathway. Tetrahedron Lett. 39, 521-524.

Milborrow, B.V., and Lee, H.S. (1998) Endogenous biosynthetic precursors of $(+)$ abscisic acid. VI. Carotenoids and abscisic acid formed by the nonmevalonate triose-pyruvate pathway in chloroplasts. Aust. J. Plant. Physiol. 25, 507-512.

Moehs, C.P., Tian, L., Osteryoung, K.W., and Dellapenna, D. (2001) Analysis of carotenoid biosynthetic gene expression during marigold petal development. Plant Mol. Biol. 45, 281-293.
Ohyanagi, H., Tanaka, T., Sakai, H., Shigemoto, Y., Yamaguchi, K., Habara, T., Fujii, Y., Antonio, B.A., Nagamura, Y., Imanishi, T., Ikeo, K., Itoh, T., Gojobori, T., and Sasaki, T. (2006) The Rice Annotation Project Database (RAP-DB): hub for Oryza sativa ssp. japonica genome information. Nucleic Acids Res. 34, D741-D744.

Page, J.E., Hause, G., Raschke, M., Gao, W., Schmidt, J., Zenk, M.H., and Kutchan, T.M. (2004) Functional Analysis of the Final Steps of the 1-Deoxy-D-xylulose 5-phosphate (DXP) Pathway to Isoprenoids in Plants Using Virus-Induced Gene Silencing. Plant Physiol. 134, 1401-1413.

Phillips, M.A., Walter, M.H., Ralph, S.G., Dabrowska, P., Luck, K., Uros, E.M., Boland, W., Strack, D., RodriguezConcepcion, M., Bohlmann, J., and Gershenzon, J. (2007) Functional identification and differential expression of 1-deoxy-D-xylulose 5-phosphate synthase in induced terpenoid resin formation of Norway spruce (Picea abies) Plant Mol. Biol. 65, 243-257.

Rieder, C.H., Jaun, B., and Arigoni, D. (2000) On the early steps of cineol biosynthesis in Eucalyptus globules. Helv. Chim. Acta. 83, 2504-2513.

Rodriguez-Concepcion, M., Ahumada, I., Diez-Juez, E., Sauret-Gueto, S., Lois, L.M., Gallego, F., CarreteroPaulet, L., Campos, N., and Boronat, A. (2001) 1-Deoxy-D-xylulose 5-phosphate reductoisomerase and plastid isoprenoid biosynthesis during tomato fruit ripening. Plant J. 27, 213-222.

Rodrıguez-Concepcion, M., and Boronat, A. (2002) Elucidation of the Methylerythritol Phosphate Pathway for Isoprenoid Biosynthesis in Bacteria and Plastids. A Metabolic Milestone Achieved through Genomics. Plant Physiol. 130, 1079-1089.

Rohdich, F., Wungsintaweekul, J., Luttgen, H., Fischer, M., Eisenreich, W., Schuhr, C.A., Fellermeier, M., Schramek, N., Zenk, M.H., and Bacher, A. (2000) Biosynthesis of terpenoids: 4-diphosphocytidyl-2-Cmethyl-D-erythritol kinase from tomato. Proc. Natl. Acad. Sci. U.S.A. 97, 8251-8256.

Rohmer, M. (2003) Mevalonate-independent methylerythritol phosphate pathway for isoprenoid biosynthesis. Elucidation and distribution. Pure Appl. Chem. 75, 375-387.

Rohmer, M. (1999) The discovery of a mevalonate independent pathway for isoprenoid biosynthesis in bacteria, algae and higher plants. Nat. Prod. Rep. 16, 565-574.

Sagner, S., Eisenreich, W., Fellermeier, M., Latzel, C., Bacher, A., and Zenk, M.H. (1998) Biosynthesis of 2-C-methyl-D-erythritol in plants by rearrangement of the terpenoid precursor, 1-deoxy-D-xylulose-5phosphate. Tetrahedron Lett. 39, 2091-2094.

Sasaki, T., and Matsumoto, T. (2002) The genome sequence and structure of rice chromosome 1. Nature 420, 312-316.

Sato, S., Nakamura, Y., Kaneko, T., Katoh, T., Asamizu, E., and Tabata, S. (2000) Structural analysis of Arabidopsis thaliana chromosome 3. I. Sequence features of the regions of $4,504,864 \mathrm{bp}$ covered by sixty $\mathrm{P} 1$ and TAC clones. DNA Res. 7, 131-135. 
Schwender, J., Muller, C., Zeidler, J., and Lichtenthaler, H.K. (1999) Cloning and heterologous expression of a cDNA encoding 1-deoxy-D-xylulose-5-phosphate reductoisomerase of Arabidopsis thaliana. FEBS Lett. 455, 140-144.

Scolink, P.A. and Bartley, G.E. (1996) A table of cloned genes involved in Isoprenoid biosynthesis. Plant Mol. Biol. Report 14, 305-319.

Seetang-Nun, Y., Sharkey, T.D., and Suvachittanont, W. (2008) Isolation and characterization of two distinct classes of DXS genes in Hevea brasiliensis. DNA Seq. 19, 291-300.

Seetang-Nun, Y., Sharkey, T.D., and Suvachittanont, W. (2008) Molecular cloning and characterization of two cDNAs encoding 1-deoxy-D-xylulose 5-phosphate reductoisomerase from Hevea brasiliensis. J. Plant Physiol. 165, 991-1002.

Seki, M., lida, K., Satou, M., Sakurai, T., Akiyama, K., Ishida, J., Nakajima, M., Enju, A., Kamiya, A., Narusaka, M., Carninci, P., Kawai,J., Hayashizaki,Y., and Shinozaki, K. (2002). Arabidopsis thaliana fulllength cDNA. Published Only in Database. http://www. ncbi.nlm.nih.gov/entrez/viewer.fcgi.

Sharkey, T.D., Yeh, S., Wiberley, A.E., Falbel, T.G., Gong, D., and Fernandez, D.E. (2005) Evolution of the Isoprene Biosynthetic Pathway in Kudzu. Plant Physiol. 137, 700-712.

Sprenger G.A. (1996) Carbohydrate metabolism in Zymomonas mobilis: a catabolic highway with some scenic routes. FEMS Microbiol. Lett. 145, 301-307.

Steliopoulos, P., Wust, M., Adam, K.P., and Mosandl, A. (2002) Biosynthesis of the sesquiterpene germacrene $\mathrm{D}$ in Solidago canadensis: $13 \mathrm{C}$ and $2 \mathrm{H}$ lebelling studies. Phytochemistry 60, 13-20.

Thiel, R., and Adam, K.P. (2002) Incorporation of [1-13C]1-deoxy-D-xylulose into isoprenoids of the liverwort Conocephalum conicum. Phytochemistry 59, 269-274.

Totte, N.M.L.C., Van den Ende, W., Van Damme, E.J.M., Compernolle, F., Baboeuf, I., and Geuns, J.M.C. (2003) Cloning and heterologous expression of early genes in gibberellin and steviol biosynthesis via the methylerythritol phosphate pathway in Stevia rebaudiana. Plant Physiol. 81, 517-522.

Veau, B., Courtois, M., Oudin, A., Chenieux, J.C., Rideau, M., and Clastre, M. (2000) Cloning and expression of cDNAs encoding two enzymes of the MEP pathway in Catharanthus roseus. Biochem. Bioph. Acta. 1517, 159-163.

Verpoorte, R., and Memelink, J. (2002) Engineering secondary metabolite production in plants. Curr. Opin. Biotechnol. 13, 181-187.

Wu, S.J., Shi, M., and Wu, J.Y. (2009) Cloning and characterization of 1-deoxy-D-xylulose 5-phosphate reductoisomerase gene for diterpenoid tanshinone biosynthesis in Salvia miltiorrhiza hairy roots. Biotechnol. Appl. Biochem. 52, 89-95.

Wungsintaweekul, J., Sirisuntipong, T., Kongduang, D., Losuphanporn, T., Ounaroon, A., Tansakul, P., and DeEknamkul, W. (2008) Transcription profiles analysis of genes encoding 1-deoxy-D-xylulose 5-phosphate synthase and 2C-methyl-D-erythritol 4-phosphate synthase in plaunotol biosynthesis from Croton stellatopilosus. Biol. Pharm. Bull. 31, 852-856.

Yao, H., Gong, Y., Zuo, K., Ling, H., Qiu, C., Zhang, F., Wang, Y., Pi, Y., Liu, X., Sun, X., and Tang, K. (2008) Molecular cloning, expression profiling and functional analysis of a DXR gene encoding 1-deoxy-D-xylulose 5 phosphate reductoisomerase from Camptotheca acuminate. J. Plant Physiol. 165, 203-213.

Yu, J., Wang, J., Lin, W., Li, S., and Li, H. (2005) The Genomes of Oryza sativa: A History of Duplications. PLoS. Biol. 3, E38-E42.

Zeidler, J.G., Lichtenthaler, H.K., May, H.U., and Lichtenthaler, F.W. (1997) Is isoprene emitted by plants synthesized via a novel isopentenyl pyrophosphate pathway? Z. Naturforsch. 52C, 15-23.

Zeidler, J.G., Schwender, J., Muller, C., Wiesner, J., Weidemeyer, C., Beck, E., Jomaa, H., and Lichtenthaler, H.K. (1998) Inhibition of the non-mevalonate 1-deoxyD-xylulose-5-phosphate pathway of plant isoprenoid biosynthesis by fosmidomycin. Z. Naturforsch. 53C, 980-986. 


\section{Further Reading}

Caister Academic Press is a leading academic publisher of advanced texts in microbiology, molecular biology and medical research. Full details of all our publications at caister.com

- MALDI-TOF Mass Spectrometry in Microbiology Edited by: M Kostrzewa, S Schubert (2016) www.caister.com/malditof

- Aspergillus and Penicillium in the Post-genomic Era Edited by: RP Vries, IB Gelber, MR Andersen (2016) www.caister.com/aspergillus2

- The Bacteriocins: Current Knowledge and Future Prospects Edited by: RL Dorit, SM Roy, MA Riley (2016)

www.caister.com/bacteriocins

- Omics in Plant Disease Resistance Edited by: V Bhadauria (2016) www.caister.com/opd

- Acidophiles: Life in Extremely Acidic Environments Edited by: R Quatrini, DB Johnson (2016) www.caister.com/acidophiles

- Climate Change and Microbial Ecology: Current Research and Future Trend

Edited by: J Marxsen (2016)

www.caister.com/climate

- Biofilms in Bioremediation: Current Research and Emerging Technologies

Edited by: G Lear (2016)

www.caister.com/biorem

- Microalgae: Current Research and Applications Edited by: MN Tsaloglou (2016) www.caister.com/microalgae

- Gas Plasma Sterilization in Microbiology: Theory, Applications, Pitfalls and New Perspectives Edited by: H Shintani, A Sakudo (2016) www.caister.com/gasplasma

- Virus Evolution: Current Research and Future Directions Edited by: SC Weaver, M Denison, M Roossinck, et al. (2016) www.caister.com/virusevol

- Arboviruses: Molecular Biology, Evolution and Control Edited by: N Vasilakis, DJ Gubler (2016) www.caister.com/arbo

- Shigella: Molecular and Cellular Biology Edited by: WD Picking, WL Picking (2016) www.caister.com/shigella

-Aquatic Biofilms: Ecology, Water Quality and Wastewater Treatment

Edited by: AM Romaní, H Guasch, MD Balaguer (2016)

www.caister.com/aquaticbiofilms

- Alphaviruses: Current Biology

Edited by: S Mahalingam, L Herrero, B Herring (2016)

www.caister.com/alpha

- Thermophilic Microorganisms

Edited by: F Li (2015)

www.caister.com/thermophile
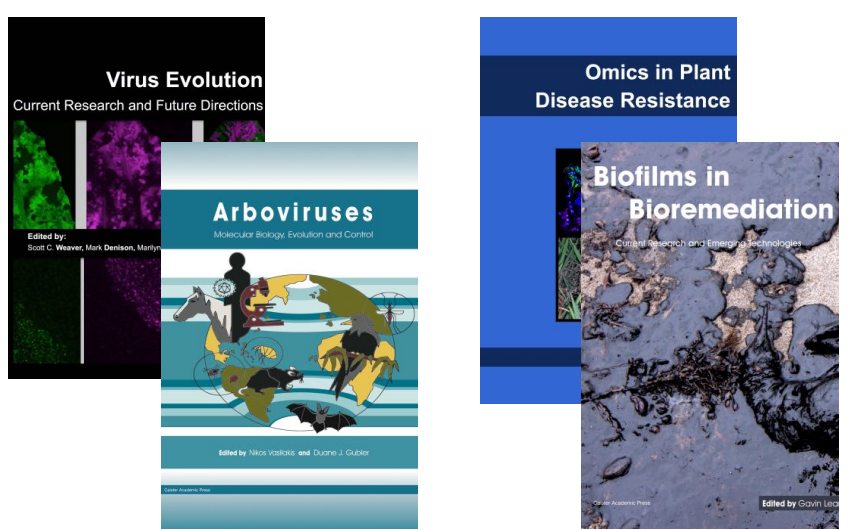
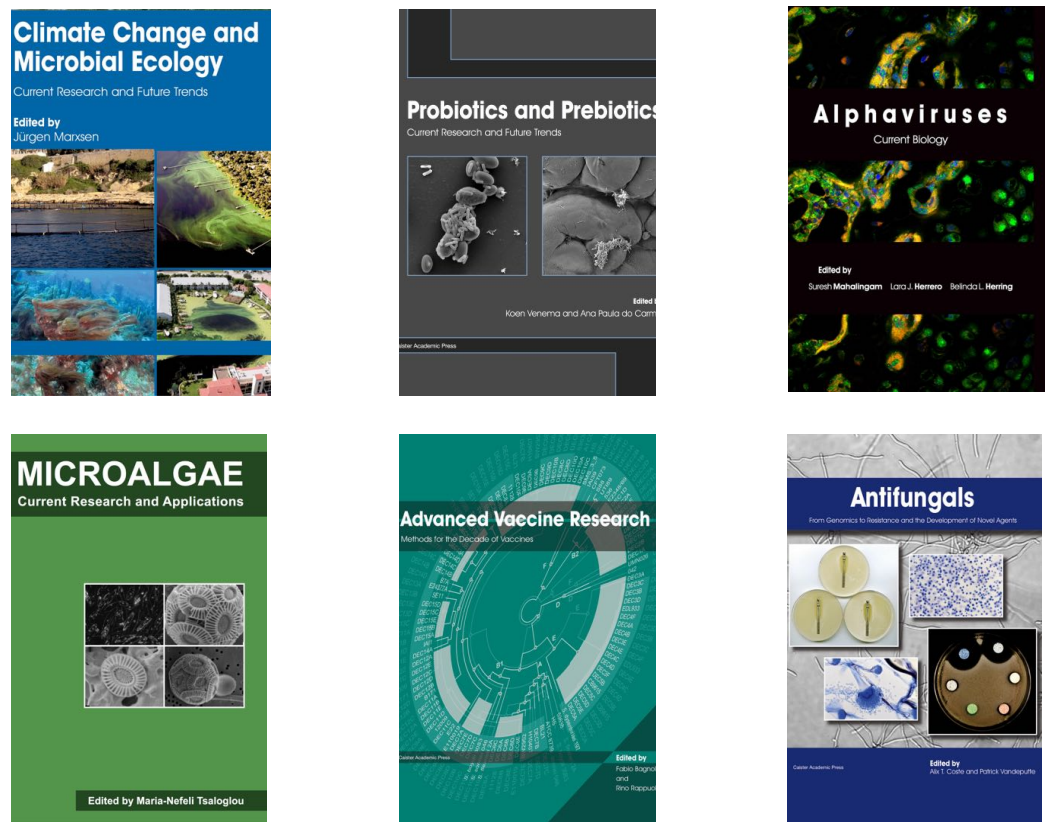

- Flow Cytometry in Microbiology: Technology and Applications Edited by: MG Wilkinson (2015) www.caister.com/flow

- Probiotics and Prebiotics: Current Research and Future Trends Edited by: K Venema, AP Carmo (2015) www.caister.com/probiotics

- Epigenetics: Current Research and Emerging Trends Edited by: BP Chadwick (2015) www.caister.com/epigenetics2015

- Corynebacterium glutamicum: From Systems Biology to Biotechnological Applications

Edited by: A Burkovski (2015)

www.caister.com/cory2

- Advanced Vaccine Research Methods for the Decade of Vaccines

Edited by: F Bagnoli, R Rappuoli (2015)

www.caister.com/vaccines

- Antifungals: From Genomics to Resistance and the Development of Novel Agents

Edited by: AT Coste, P Vandeputte (2015)

www.caister.com/antifungals

- Bacteria-Plant Interactions: Advanced Research and Future Trends Edited by: J Murillo, BA Vinatzer, RW Jackson, et al. (2015) www.caister.com/bacteria-plant

\section{- Aeromonas}

Edited by: J Graf (2015)

www.caister.com/aeromonas

- Antibiotics: Current Innovations and Future Trends

Edited by: S Sánchez, AL Demain (2015)

www.caister.com/antibiotics

- Leishmania: Current Biology and Contro Edited by: S Adak, R Datta (2015) www.caister.com/leish2

- Acanthamoeba: Biology and Pathogenesis (2nd edition) Author: NA Khan (2015)

www.caister.com/acanthamoeba2

- Microarrays: Current Technology, Innovations and Applications Edited by: Z He (2014)

www.caister.com/microarrays2

- Metagenomics of the Microbial Nitrogen Cycle: Theory, Methods and Applications

Edited by: D Marco (2014)

www.caister.com/n2 